given to who is actually going to provide this education. Dr Brown et al allude to potential problems when they say that adequate planning and fair warning has to be given to trainers and trainees about the intended changes, but it remains unclear whether these can be achieved in a climate of continuous recruitment problems in psychiatry. As a senior house officer scheme organiser, trainer and honorary lecturer I am also aware of the time constraints, which already limit the amount of time that consultants can spend with their trainees. Moreover, the new shift systems have significantly reduced the amount of time for consultants and trainees to meet. The proposals, as outlined in the article, emphasise more modular and assessment-based teaching, which in turn will inevitably require much more time devoted to trainees by their trainers. It is absolutely vital that before we embark on such significant changes we make sure that they can actually be delivered on the ground, which I very much doubt is possible with current staffing levels.

P. Lepping Consultant Psychiatrist/Honorary Lecturer, LlwynY Groes Psychiatric Unit, North East Wales NHS Trust, Wrexham Maelor Hospital, Croesnewydd Road, Wrexham LL13 7TD, e-mail: PETER.LEPPING@new-tr.wales.nhs.uk

\section{Violence risk assessment}

Dr Maden's editorial (Psychiatric Bulletin, April 2005, 29, 121-122) in response to the article by Higgins et al (Psychiatric Bulletin, April 2005, 29, 131-133) neglects a fundamental aspect of risk assessment and his recommendations therefore need to be treated with caution. Higgins et al refer to the different contexts in which risk assessment forms were being used, but Dr Maden does not appear to have taken this fully into account in advocating the routine use of the Historical/Clinical Risk-20 (HCR-20) in adult general psychiatry.

There is no doubt that the HCR-20 is a useful tool in forensic settings, where it is already widely used. However, there would be significant time and cost implications to introducing it routinely into general adult settings, and it could be argued that this would not be sensible or cost-effective. Although Dr Maden starts by advocating 'a more systemic approach in marginal cases' which it is hard to disagree with, his advocacy of the routine use of the 'ideal' HCR-20 does not appear to address whether this is a feasible option with non-marginal cases. The HCR-20 can be very effective in supporting teams in assessing and reviewing ongoing risks, but it is not clear that it is a practical solution to supporting, for example, the risk assessment and management decisions of a junior psychiatrist doing an assessment of a new patient in an accident and emergency department in the middle of the night.

Dr Maden notes that Higgins et al reported that many consultants did not attend the (presumably free) half-day training on violence risk assessment already on offer within their services. To suggest 3-day external fee-based training specifically for violence risk assessment seems a disproportionate response when other priorities in mental health (for example assessing the risk of self-harm) are not identified as requiring such expensive formalised training.

It is a pity that neither Higgins et al nor Dr Maden were able to expand on the possible utility of the CARDS project (Watts et al, 2004) that the study of Higgins et al was part of. This appears to be a worthwhile collaborative attempt at developing a more standardised approach to risk assessment in general adult psychiatry. It is also free and potentially more easily integrated into routine clinical practice than the wholesale use of the HCR-20.

WATTS, D., BINDMAN, J., SLADE, M., et al (2004) Clinical assessment of risk decision support (CARDS): The development and evaluation of a feasible violence risk assessment for routine psychiatric practice. Journal of Mental Health, 13, 569-581.

Dan Beales Specialist Registrar in Forensic Psychotherapy, The Red House, Bolton, Salford and Trafford Mental Health NHS Trust, 78 Swinton Road, Salford M27 8GB, e-mail: Daniel.Beales@ edenfield.bstmht.nhs.uk

\section{Clinical excellence awards}

Having once more been through the annual awards process of reviewing citations and CVs, within both trust and College systems, we are writing to express our sense of disillusionment and distaste at the whole rigmarole. Not only is it extremely time-consuming for all involved, especially the applicants, but it is intrinsically unreliable and demeaning as a method of enhancing doctors' pay. The changes in the system from $A, B, C$ to clinical excellence awards and various precious metals have been accompanied by a series of 'domains' that overlap remarkably and for which we have yet to see an agreed model criterion. How does one assess whether an individual doctor is 'delivering a high quality service' or 'managing a high quality service'? What is the definition of a high quality service?

A doctor working overtime because of the poor quality of the service that he or she is involved in perhaps should be preferentially rewarded for staying there rather than going to an easier place. Is 'high quality' defined as patient outcome, for example how many patients with depression are cured or surgical operations carried out without complaint or side-effects, or is it because the service is carried out in accordance with the wishes of the trust, strategic health authority or government? Carrying out an audit or introducing a 'modernised' style of service are automatically noted as positive, but continuing to see difficult patients who complain, don't get better and generate 'untoward incidents' may even downgrade you in your chief executive's eyes.

As with the previous system, the busier and more active the doctors are in seeing patients and providing a comprehensive service - usually beyond contracted hours in the case of many general adult psychiatrists - the less likely they are to be able to sit down and fill out the form with sufficient details of committees or working groups attended, papers published or lectures given. What are termed 'clinical excellence awards' are really awards for clerical excellence. Most of the information is entirely impossible to check, particularly since we do not have a routine patient feedback system (as they do in the USA) or any generally valid outcome measures.

However, even after having created time to fill in the forms, the clinician seeking a national award is then faced with a cruel timetable of waiting until November to find out if an award has been offered and, if not, then having to re-scramble the whole application over Christmas. This is a very difficult time for committees to meet in order to comply with the narrow timetable for submission before the end of January. Once the forms are despatched, the processes and decision-making of the higher Advisory Committee on Clinical Excellence Awards committee levels (i.e. beyond trusts and colleges) is so obscure as to be postKafkaesque. Again, it is likely that these committees and assessors have even less knowledge of the realities behind the forms and will be faced by hundreds of CVs. How can they deal with variations in specialty, age, gender and ignorance of the true resources or quality of care in any given trust?

At the local level matters are even more delicate, small amounts of money per point gained contrasting with the substantial demoralisation following rejection. Many trusts have operated an every second year policy, but is this consistent nationwide? Why not only try and pull your weight every other year? The process becomes essentially an alluring form of salary redistribution that conveniently hides a ceiling on salaries, since the chance of progressing nationally, beyond level 8 , remains less than $10 \%$.

Our view is that these embarrassing and essentially uncertain processes should be abandoned and that the consultant pay scale should be extended into areas of 
incremental seniority via standardised reviews based on the appraisal process. Committee and managerial work should be allocated additional sessions, openly assessed and competed for and again acknowledged via seniority. Loyalty of service - it takes years to generate an effective knowledge of locality and networks - should also be acknowledged to counter the disheartening spectacle of locums being paid more than committed 'regulars'. We admire the dedication and care of all those involved in trying to help develop scoring and assessment systems for these awards, but our own view remains that the aftermath is always one of a sense of embarrassment, depression and pervasive ennui.

*TrevorTurner Consultant Psychiatrist, Clinical Director, Homerton University Hospital,

Michael Maier Senior Lecturer, Honorary Consultant Psychiatrist, West London Mental Health NHS Trust, Uxbridge Road, Southall, Middlesex UB1 3EU

\section{Venlafaxine discontinuation symptoms on prolonged low dose}

The National Institute for Clinical Excellence guidelines state that all patients prescribed antidepressants should be informed that, although the drugs are not associated with tolerance and craving, discontinuation/withdrawal symptoms may occur on stopping, missing doses or occasionally, on reducing the dose of the drug. These symptoms are usually mild and self-limiting, but can occasionally be severe, particularly if the drug is stopped abruptly.

Discontinuation symptoms are commonly described in patients taking venlafaxine (Pinzani et al, 2000; Baboolal,
2004). Shock-like sensation has been described with the discontinuation of venlafaxine (Reeves et al, 2003) and more often with the discontinuation of selective serotonin reuptake inhibitors (SSRIs) (Black et al, 2000). This case differs from others because the patient was taking a sub-therapeutic dose and the duration of the discontinuation symptoms was longer.

A 41-year-old research scientist with a 15-year history of bipolar affective disorder has been treated successfully with $600 \mathrm{mg}$ lithium and venlafaxine since 1995 when he had a manic episode followed by a depressive episode. He has adhered well to his repeat prescriptions and he has remained euthymic. His lithium blood levels have remained stable and within the therapeutic range.

After 5 years in remission the dose was decreased to $50 \mathrm{mg}$ without any problems, but when trying to stop it he would always experience discontinuation symptoms hours after missing the dose. Five years later it was decided not to issue the prescription because the dose was no longer available.

From the morning after missing his usual night-time dose, he started to experience shock-like sensations, described as 'electric shocks with a loud noise in my brain', every few minutes, irritability, emotional lability, poor concentration, dyskinesia in the form of sudden uncontrollable body movements, dry mouth, 'tannin-like' taste in the mouth, profuse sweating and insomnia. He was unable to work or drive. The symptoms improved on the fifth day, but continuing poor concentration and shock-like sensations rendered him unable to resume his normal activities. On the tenth day, his concentration was still poor and the shock-like sensations were occurring a few times a day. By then his emotional lability had resolved but the dyskinesia persisted. From the tenth day all remaining symptoms resolved with a transient recurrence of electric shock sensations up to day 17 . He was then able to return to work and to drive. He has remained well since.

Discontinuation symptoms with venlafaxine are similar to those with SSRIs and it is very likely that the serotonin transmitter system is involved. A UK survey (Young \& Currie, 1997) has shown that too few consultant psychiatrists and general practitioners are aware of discontinuation symptoms of antidepressants. Venlafaxine should be gradually tapered and the patient should be informed of the potential physical and psychological disturbances abrupt discontinuation can cause.

BABOOLAL, N. S. (2004) Venlafaxine withdrawal syndrome: report of seven cases inTrinidad. Journal of Clinical Psychopharmacology, 24, 229-231.

BLACK, K., SHEA, C., DURSUN, S., et al (2000) Selective serotonin reuptake inhibitor discontinuation syndrome: proposed diagnostic criteria. Journal of Psychiatry and Neuroscience, 25, 255-261.

PINZANI, V., GINIES, E., ROBERT, L., et al (2000) Venlafaxine withdrawal syndrome: report of six cases and review of the literature. Revue de Médecine Interne, 21, 282-284.

REEVES, R. R., MACK, J. E. \& BEDDINGFIELD, J. J. (2003) Shock-like sensations during venlafaxine withdrawal. Pharmacotherapy, 23, 678-681.

YOUNG, A. H. \& CURRIE, A. (1997) Physicians knowledge of antidepressant withdrawal effects: a survey. Journal of Clinical Psychiatry, 58 (suppl. 7), $28-30$

Sophie Dechene Senior House Officer, Merseycare, Liverpool, e-mail: sdechene@ doctors.net.uk 